\title{
Control of CXCR2 activity through its ubiquitination on $\mathrm{K} 327$ residue
}

\author{
Héloise M Leclair ${ }^{1,2,3}$, Sonia M Dubois ${ }^{1,2,3}$, Sandy Azzi ${ }^{1,2,3}$, Julie Dwyer ${ }^{1,2,3}$, Nicolas Bidère ${ }^{1,2,3}$ and Julie Gavard ${ }^{1,2,3,4^{*}}$
}

\begin{abstract}
Background: The interleukin-8 chemokine (IL-8) G-protein coupled receptor CXCR2 governs pro-inflammatory and pro-angiogenic responses in leukocytes and endothelial cells. At a molecular standpoint, CXCR2 is widely reported to operate through calcium flux, phosphoinoisitide 3 kinase (PI3K) and mitogen-activated protein kinase (MAPK). While CXCR2 trafficking is suspected to be intertwined with its signaling, the exact mechanism is not fully elucidated.

Results: Here, we identified the lysine 327 within the CXCR2 C-terminal tail as a key residue for ubiquitination, internalization, and signaling. First, the substitution to an arginine of $\mathrm{K} 327$ mutation was associated with a reduction in CXCR2 poly-ubiquitination. While WT CXCR2 was rapidly internalized following IL-8 administration, K327R mutant remained at the plasma membrane. Finally, K327R mutant failed to promote the recruitment of $\beta$-arrestin2, as estimated by imagery and bioluminescence resonance transfer. As a consequence, the activation of intracellular signaling, including both early events such as ERK phosphorylation and the increase in calcium flux, and the latter activation of the AP1 and NF-KB transcription factors, was blunted.
\end{abstract}

Conclusions: Overall, our results demonstrate that CXCR2 ubiquitination on K327 residue modulates agonist-activated CXCR2 cell sorting and intracellular signaling. Thus, the inhibition of K327 ubiquitination might emerge as an effective mean to curb exacerbated CXCR2 signaling in several pathological conditions, such as inflammatory diseases and cancer.

Keywords: Chemokine receptor, Ubiquitination, Arrestin, Internalization, Signaling

\section{Background}

CXCR2 is a seven transmembrane G-protein coupled receptor (GPCR) for the ELR + CXCL8 (IL-8) chemokine that transduces pro-angiogenic and pro-inflammatory responses in endothelial and immune cells [1-5]. Indeed, IL-8/CXCR2 signaling axis plays multiple functions in the course of physiological and pathological neo-vessel formation. Likewise, IL-8 signaling is instrumental in leukocyte migration [6]. Notably, it does so by stimulating endothelial cell proliferation, permeability, and migration, and by attracting lymphocytes, macrophages, and neutrophils to perivascular regions. For instance, we recently demonstrated that IL-8 operates through CXCR2 and phosphoinositide 3-kinase $\gamma(\mathrm{PI} 3 \mathrm{~K} \gamma$ ) to promote angiogenesis and macrophage accumulation in retina, while curbing the endothelial barrier function [3]. Importantly, interfering

\footnotetext{
* Correspondence: Julie.gavard@inserm.fr

'CNRS, UMR8104, 22 rue Mechain, 75014 Paris, France

${ }^{2}$ INSERM, U1016, 22 rue Mechain, 75014 Paris, France

Full list of author information is available at the end of the article
}

with this pathway quelled laser-induced vascular dysfunctions in mouse retina. Similar observations were done in the context of cancers, including malignant brain tumors, prostate tumors, pancreatic ductal adenocarcinoma, chemically induced-skin tumors, Ras-mediated tumors and inflammation-driven tumors [2,7-10]. These data place CXCR2 as a promising pharmacological target in many human diseases and pathological conditions [4]. In that view, targeting GPCR constitutes the primary option for pharmacological intervention and drug development [11]. However, many of these compounds act as direct antagonists leaving uncertain their ability to target pathways that are aberrantly and/or constitutively active.

Physiologically, GPCR signaling can be either twisted or interrupted by intracellular cascades implying endocytosis and ubiquitination [12]. Similarly to the CXCL12 (also known as stromal derived factor $1 \alpha, \mathrm{SDF} 1 \alpha)$ chemokine receptor CXCR4, ligand-activated CXCR2 undergoes endocytosis in clathrin-coated vesicles [13,14]. In that view, the viral CXCR2 homolog vGPCR from the Kaposi 
Sarcoma Herpes Virus, harbors a consensus adaptin 2 (AP2) binding motif in its C-terminal tail, which drives its constitutive shuttling in clathrin-coated vesicles, and further adjusts its signaling activity [15]. Moreover, CXCR2 internalization might involve scaffold proteins, including $\beta$-arrestins, AP2, actin-binding proteins and yet to be determined PDZ ligand motif binding proteins [13,14,16-19]. However, how exactly CXCR2 signaling, intracellular sorting and ubiquitination are coordinated is not fully elucidated. This prompted us to explore the molecular contribution of lysine residues, operating as ubiquitin acceptors, to CXCR2 activity.

\section{Results and discussion}

The ligation of G-protein coupled receptor CXCR2 drives its relocation into endosomal compartments, where it could be targeted for lysosomal degradation [13]. This dynamic process has been shown to rely on specific motifs, including lysine residues within the C-terminal domain [13]. To investigate which lysine could contribute to the fine-tuning of CXCR2 trafficking, we first proceed to amino-acid sequence alignment between residues 316 and 347 on the human sequence. Our in silico analysis identified two lysine residues on position 327 and 337 in the human sequence, conserved in pig, mouse, and chicken, and shared between CXCR2 and CXCR1 (Figure 1a). As expected, structural prediction did not reveal major changes in the overall CXCR2 molecular organization in the context of K-R mutations (Figure 1b). Point mutation in each of these two lysines was thus engineered on the full-length (FL) receptor, harboring an AU5 tag. The expression of K327R (named R1) and K337R (named R2) FL CXCR2 mutants was found similar to the one of wild type (WT) when transfected in HEK-293T cells (Figure 1c). These constructs have been thus used throughout the study. Because lysine residues functions as ubiquitin-acceptors, we next setup the experimental conditions to visualize CXCR2 ubiquitination, using validated methods for intracellular targets [20,21]. CXCR2 immunoprecipitated fractions were analyzed under denaturing conditions, ie 1\% SDS final concentration to prevent non-covalent protein interactions but without heat denaturation that could favor membrane protein aggregation. Using these conditions, the monoclonal anti-ubiquitin antibody (clone P4D1) readily detected modified CXCR2 in the high molecularweight fraction (Figure 1d). Additionally, denaturation prior immunoprecipitation dissociated putative contaminations with associated ubiquitinated partners of CXCR2, such as $\beta$-arrestins (Figure 1d). Similarly, ubiquitination addition was detected in the CXCR2 immunoprecipitated fractions from endothelial cells that endogenously expressed the receptor (Additional file 1: Figure S1). As a further control, CXCR2 antagonist
SB225002 [3,22] was sufficient to curtail CXCR2 ubiquitination in response to IL-8 (Figure 1e). Of note, CXCR2 ubiquitination species accumulated in cells pretreated with the proteasome inhibitor MG132, suggesting that ubiquitination marks CXCR2 for degradation (Figure 1f). We next examine the ubiquitination profile of K-R CXCR2 mutants. Interestingly, the typical ubiquitination ladder discerned in response to IL-8 challenge in WT CXCR2-expressing HEK-293T cells was profoundly reduced when K327 residue was substituted for an arginine (Figure 1g). Our data thus suggest that the K327 residue is critical for K-linked ubiquitination of activated CXCR2.

We next aimed at understanding how K327 could modulate CXCR2 trafficking. In this scenario, localization of K-R CXCR2 mutants was further characterized by confocal microscopy (Figure 2). In starved non-stimulated HEK-293T cells, WT CXCR2 primarily labeled plasma membrane and redistributed in internal vesicle-like dots shortly following IL-8 challenge (15 minutes, Figure 2a). By contrast, K327R CXCR2 remained accumulated at the plasma membrane regardless of IL-8 treatment. On the other hand, the K337R mutant mimicked WT CXCR2, as it shuttled from the plasma membrane to internal vesicles (Figure 2a). This was found similar to endogenous CXCR2 dynamics in IL-8-challenged THP1 monocytes (Additional file 2: Figure S2). Likewise, CXCR2 internalization, as monitored through antibody uptake assay followed by an acid wash, was enhanced in response to IL-8 administration, unless K327 was mutated (Figure 2b). Co-labeling with Rab7 and AP2, which illuminates late and early endosomes, respectively, indeed supports the notion that WT and K337R CXCR2 were endocytosed upon IL-8 stimulation (Figure 2c). Conversely, plasma membrane staining of the K327R mutant was not curbed by IL-8 treatment, unlike WT and K337R mutant (Figure 2c). Altogether, our results show that the K327R point mutation is sufficient to restrain CXCR2 internalization upon ligand binding, suggesting therefore that this residue is critical to ensure proper trafficking of the activated receptor.

We next investigated whether K327 residue participates to CXCR2 signaling ability. To this aim, one of the most proximal events in GPCR activation, namely $\beta$-arrestin2 activation was first assessed. Interestingly, K327R single substitution prevented from IL-8-induced $\beta$-arrestin2 clustering, observed normally in cells transfected with WT and K337R CXCR2 (Figure 3a-b). Likewise, K327R CXCR2 mutant less efficiently activated $\beta$-arrestin 2 in response to IL-8, as estimated through Bioluminescence Resonance Energy Transfer (BRET) technology (Figure 3c). As phosphorylation is also an important hallmark of GPCR activation upon ligation [23], we checked the status of CXCR2 tyrosine phosphorylation in immunoprecipitation experiments (Figure 4a). As expected, IL-8 drove WT 


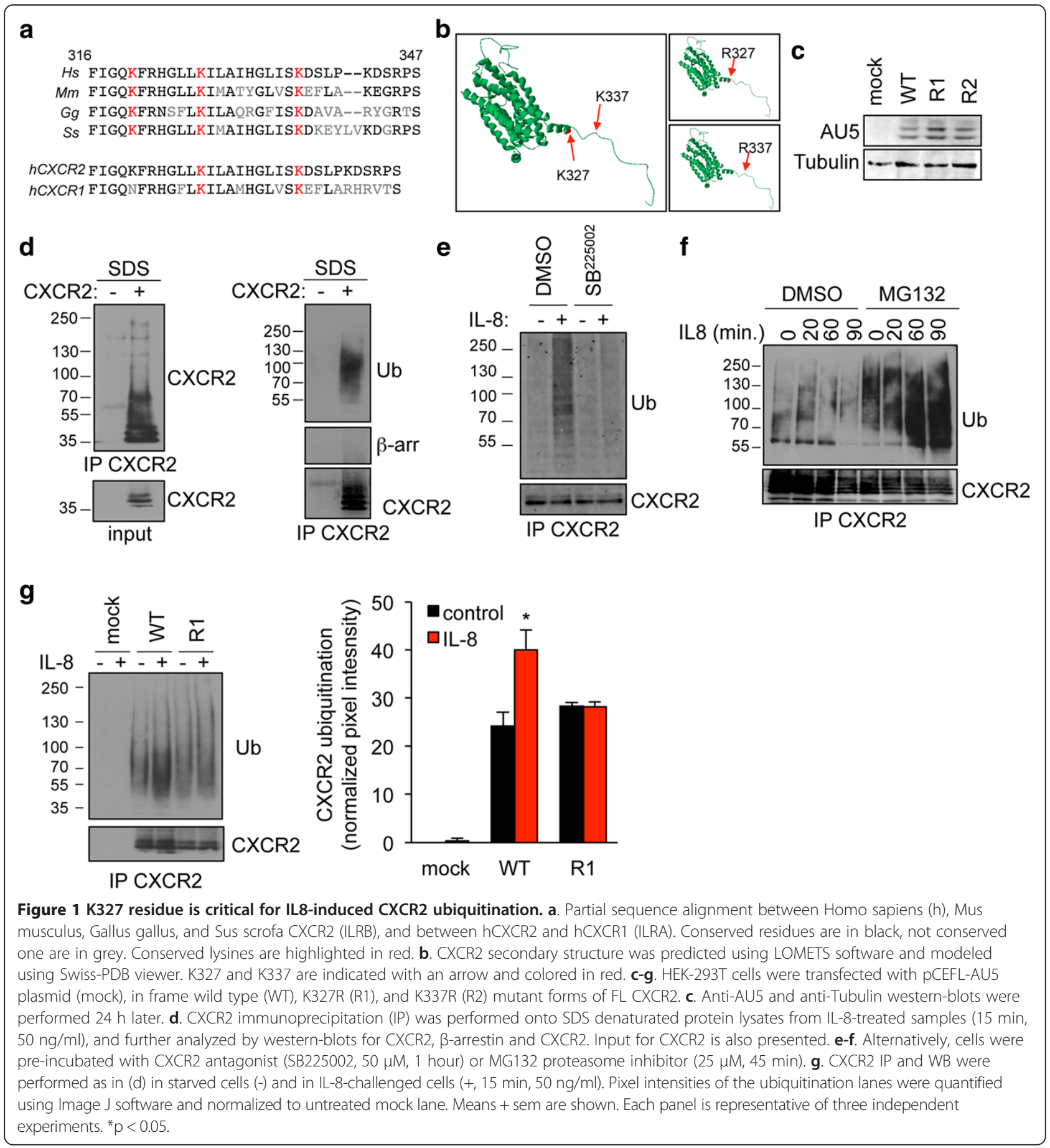

CXCR2 phosphorylation. In sharp contrast, the signal was barely detectable when K327R mutant was ectopically expressed in HEK-293T (Figure 4a). Secondly, intracellular signaling, namely extracellular regulated kinase 1/2 (ERK1/2) phosphorylation and increase in calcium influx, was evaluated in WT, K327R and K337R CXCR2 expressing HEK-293T cells (Figure 4b-c). Our data showed that IL-8 could not elicit either CXCR2 and ERK1/2 phosphorylation, or the elevation in intracellular calcium concentration in K327R CXCR2transfected cells. Furthermore, luciferase-based reporter assays unveiled that K327 mutation abrogates AP1 and NF- $\mathrm{KB}$ activation downstream of CXCR2 stimulation by IL-8 (Figure 4d). Thus, our data demonstrate that the signaling function of CXCR2 is compromised when the K327 residue is not available for ubiquitination. 


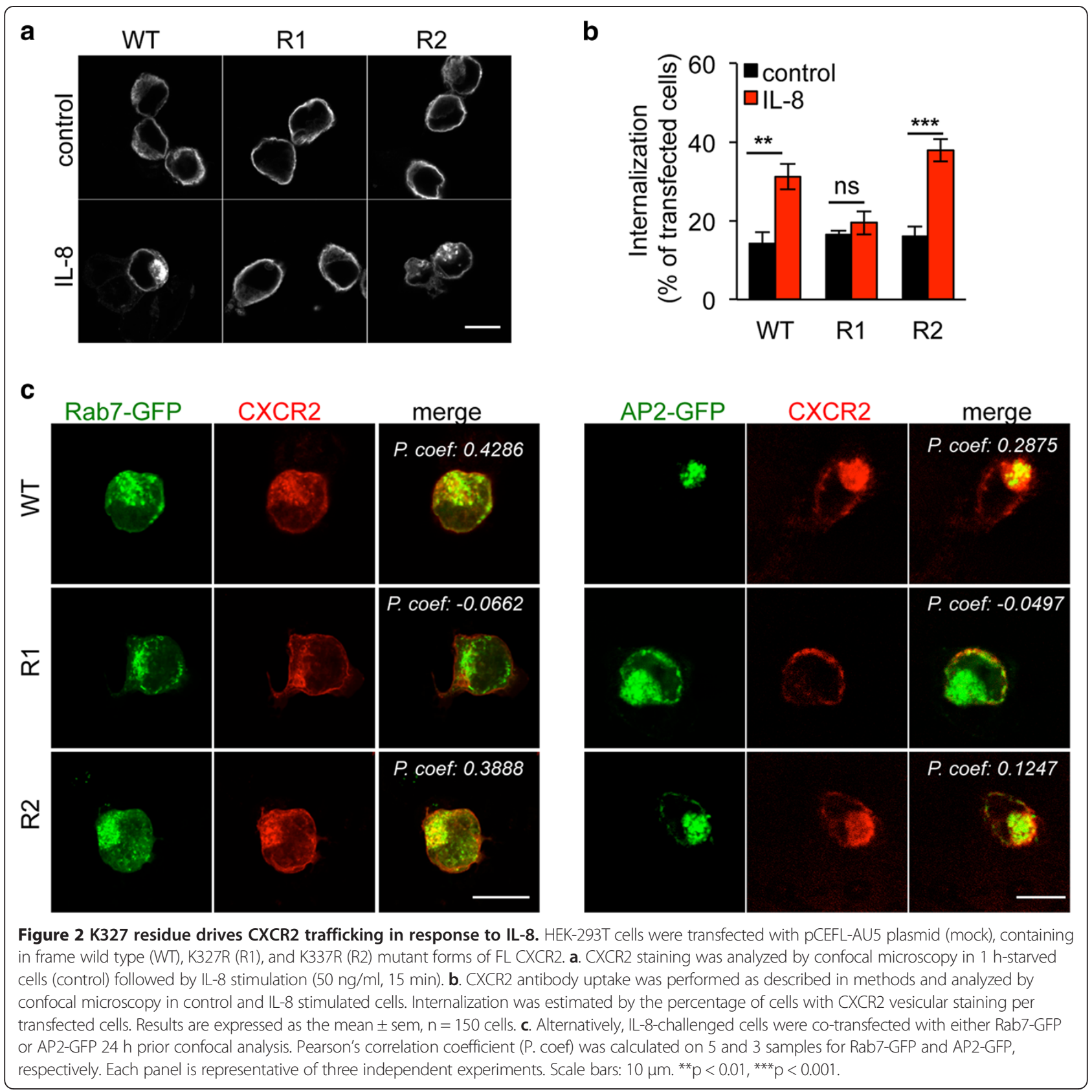

\section{Conclusions}

We report here that the conserved K327 residue on CXCR2 is a key ubiquitin acceptor upon IL-8 challenge in ectopic cell models. Of note, interfering with this post-translational modification abrogates CXCR2 trafficking and signaling. In this scenario, CXCR2 polyubiquitination, by a yet to be determined ligase, precedes $\beta$-arrestin2 recruitment and CXCR2 internalization, and is therefore placed upstream in the IL-8-mediated intracellular signaling cascade. Our data thus highlight an original option to specifically and finely tune CXCR2 signaling in pathological situations, where aberrantly elevated, such as tumor angiogenesis and inflammation.

\section{Methods}

Cell culture and transfections

HEK-293T cells (ATCC) were maintained in Dulbecco's modified Eagle's medium supplemented with $10 \%$ fetal bovine serum and antibiotics. Previously described protocols for transfection were used [24]. 

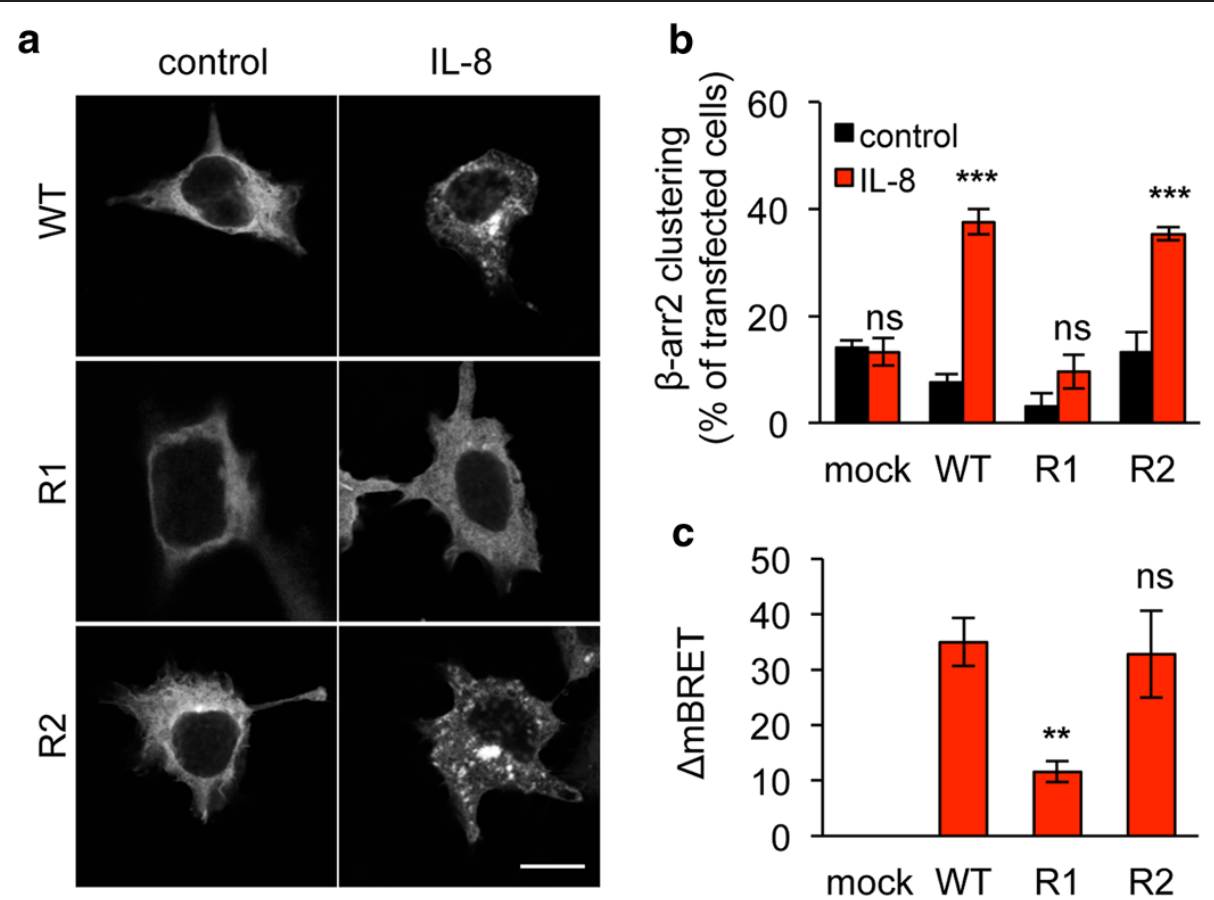

Figure 3 K327R mutation eliminates $\beta$-arrestin2 recruitment. HEK-293T cells were transfected with pCEFL-AU5 plasmid (mock), containing in frame wild type (WT), K327R (R1), and K337R (R2) mutant forms of FL CXCR2. One day later, cells were starved (control) or exposed to IL-8 (50 ng/ml, $15 \mathrm{~min}$ ). a-b. Cells were additionally transfected with $\beta$-arrestin2-GFP ( $\beta$ arr2) and the percentage of cells with clustered $\beta$-arrestin2-GFP staining was evaluated. Results are expressed as the mean \pm sem, $n=150$ cells. c. BRET analysis was performed as described in methods, $\triangle$ mBRET was calculated and the mean \pm sem is shown. Each panel is representative of three independent experiments. Scale bars: $10 \mu \mathrm{m} .{ }^{* *} p<0.01,{ }^{* * *} p<0.001$.

\section{Reagents and antibodies}

Recombinant human interleukin 8 (IL-8) was purchased from Peprotech, CXCR2 antagonist SB225002 and MG132 were from Calbiochem. The following antibodies were used: mouse and rabbit anti-CXCR2, rabbit antiERK2, mouse anti-tubulin, mouse anti-poly-ubiquitinated chains, clone P4D1 (Santa Cruz); rabbit anti-phophoERK1/2, rabbit anti- $\beta$-arrestin1/2 (Cell Signaling); mouse anti-phospho-tyr (4G10 clone, Millipore), and mouse antiAU5 (Eurogentec). AlexaFluor-conjugated antibodies were from Invitrogen.

\section{DNA constructs}

Full length (FL) K327R (R1) and K337R (R2) CXCR2 mutants were prepared from human wild type (WT) CXCR2, cloned in frame in PCEFL-AU5 expression vector [15]. pGFP- $\beta$-arrestin2 [24], EGFP- $\beta 2$-adaptin (AP2) [25] and pYFP-Rab7 were described previously [26].

\section{Structural analysis}

CXCR2 protein sequence was introduced to the LOMETS software [27] and the predicted secondary structure with the lowest Z-score was chosen for modeling, using the Swiss-PDB viewer 36 (www.expasy.org/spdbv/). Mutations were introduced using the mutation tool, according to the manufacturer's instruction.

\section{Immunofluorescence}

HEK-293T cells were grown onto glass coverslips, fixed (PBS-paraformaldheyde 4\%), permeabilized (PBS-Triton $0.5 \%$ ) and blocked (PBS-BSA 3\%). Following incubations with primary and AlexaFluor-conjugated secondary antibodies for $1 \mathrm{~h}$ in PBS-BSA 1.5\%, samples were mounted (ProLong, Invitrogen), visualized and analyzed under confocal microscopy (TCS/SP5, Leica). Pearson's coefficient was measured using the Image J plugin on 3 to 5 samples.

\section{Ubiquitination assays}

HEK-293T cells were transfected with mock, CXCR2-WT or K327R (R1) cDNA plasmids. 24 hours post-transfection, cells were treated as indicated and were further lysed (25 mM Hepes pH7.4, $130 \mathrm{mM} \mathrm{NaCl}, 10 \%$ glycerol, $8 \mathrm{mM}$ $\beta$-glycerophosphate, $0.2 \%$ Igepal, $1 \mathrm{mM} \mathrm{DTT,} 1 \mathrm{mM}$ NaVO4, $1 \mathrm{mM} \mathrm{NaF}$, protease inhibitors, $1 \%$ SDS and $1 \mu \mathrm{l}$ benzonase (250 U/ $\mu \mathrm{l}$, Novagen)) on ice. After 30 minutes, SDS was diluted to reach a final concentration of $0.1 \%$ and samples were precleared with Protein G agarose (Sigma) for $30 \mathrm{~min}$ and then incubated with antibody to CXCR2 


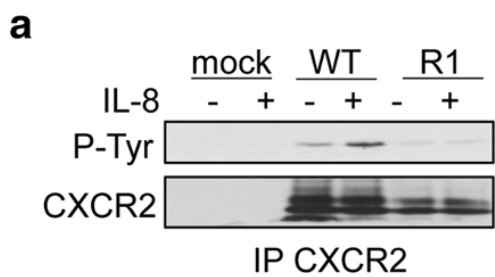

b

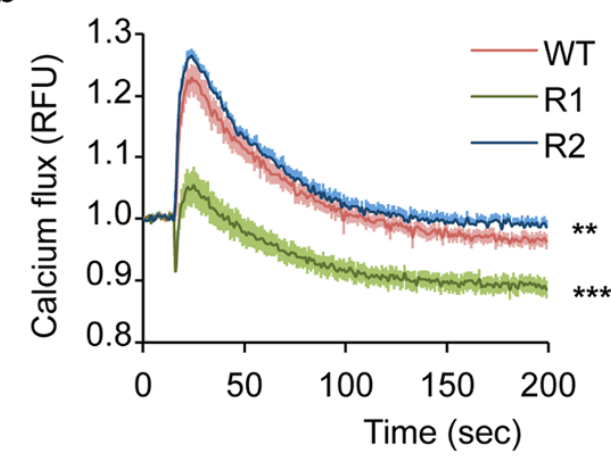

d

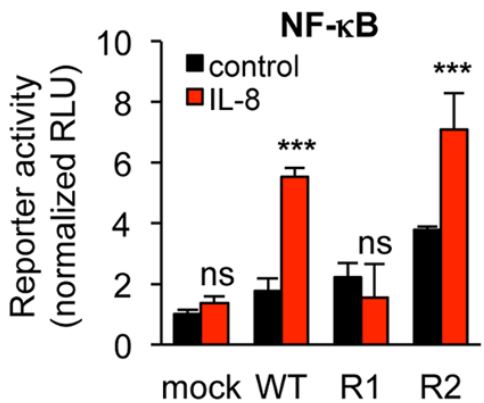

C
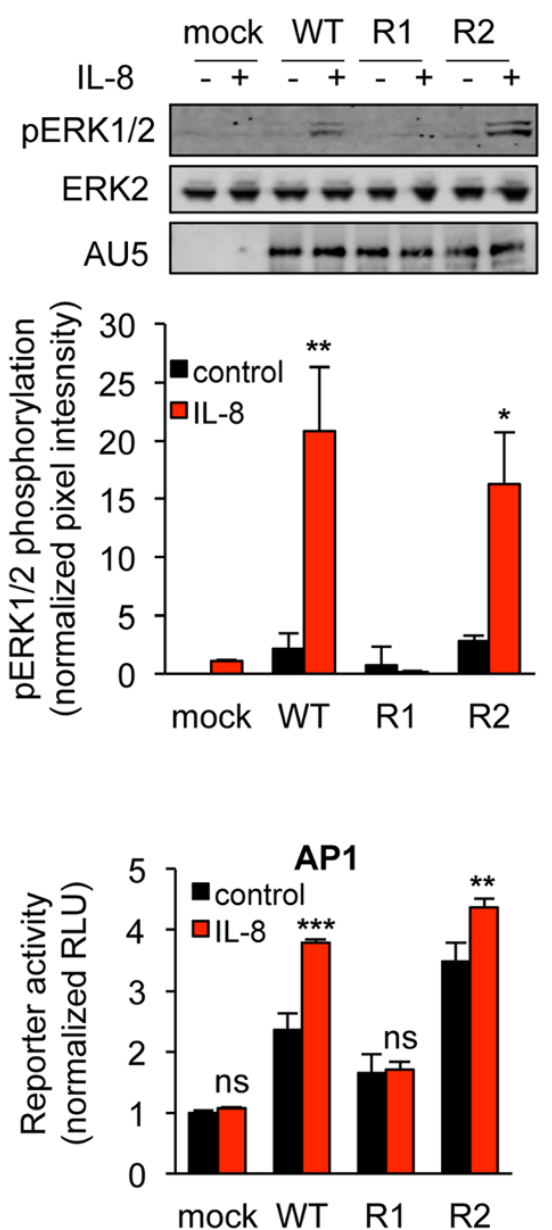

Figure 4 K327R mutation abrogates CXCR2 signaling in response to IL-8. HEK-293T cells were transfected with pCEFL-AU5 plasmid (mock), containing in frame wild type (WT), K327R (R1), and K337R (R2) mutant forms of FL CXCR2. One day later, cells were starved (control) or exposed to IL-8 (50 ng/ml, 15 min, unless specified). a. Anti-phospho-Tyr (4G10), anti-CXCR2 western-blots were performed in CXCR2 immunoprecipitated (IP) fraction. b. Calcium flux was measured using the Fluo4NW probe in response to IL-8 for the indicated times. Fluorescent units were normalized to control conditions. c. Anti-AU5, anti-pERK1/2 and anti-ERK2 western-blots were performed. Pixel intensities of the pERK1/2 lanes were quantified using Image J software and normalized to total ERK2. $\mathbf{d}$. Promoter activity of both NF-KB and AP1 was monitored through luciferase-based assays. Each panel is representative of three independent experiments. Means + sem are shown. ${ }^{* *} p<0.01,{ }^{* * *} p<0.001$.

and Protein $\mathrm{G}$ agarose for 1 hour at $4^{\circ} \mathrm{C}$. Ubiquitination status were analyzed by immunoblot using antibody against ubiquitin.

\section{Western-blots}

Protein lysates were processed for western-blots as described previously [2]. Equal amounts of protein were loaded onto 4-12\% acrylamide gradient gels (NUPAGE, Invitrogen) and transferred onto PVDF membranes (Thermo Scientific) for further analysis using the infrared scanner system (LiCOR).

\section{Cytosolic calcium measurements}

Changes in intracellular $\mathrm{Ca} 2+$ levels were assessed in HEK-293T as previously described [28], using Fluo-4 NW calcium indicators (Life Technologies), following the manufacturer's instructions. Fluorescence was read in 96-well plate format at room temperature, using appropriate settings (excitation/emission 494/516 nm, BMG microplate reader).

\section{Luciferase assays}

Firefly luciferase constructs downstream of NF- $\mathrm{kB}$ and AP1 consensus sites [2] were co-transfected with Renilla luciferase pRL-TK plasmid (Promega), together with 250 ng pCEFL-AU5-CXCR2 constructs. Following incubation with IL-8 (50 ng/ml, 6 hours), luciferase activity was analyzed as described previously [2].

\section{BRET assays}

HEK-293T cells were transfected with $0.1 \mu \mathrm{g}$ per well of the DNA construct coding for double-brilliance 
$\beta$-arrestin2 [29], together with CXCR2 constructs. Twodays after transfection, IL-8 (50 ng/ml) and DeepBlue coelenterazine substrate $(5 \mu \mathrm{M}$, Perkin Elmer) were added. Luminescence was measured (Mithras fluorescenceluminescence detector, Berthold) as previously described in [29]. The BRET signal was determined by calculating the ratio of the light emitted by the fluorescent acceptor and the light emitted by Luc. The values $(\triangle \mathrm{mBRET})$ were corrected by subtracting the background BRET signals detected in non-stimulated cells.

\section{Statistical analysis}

All data are expressed as mean + s.e.m from three independent experiments. One-way and two-way ANOVA tests with post hoc Tukey's analysis were used to assess statistical significance (Prism 6.0 GraphPad Software), and $P$ values are indicated on figures as follows: * for $\mathrm{p}<0.05$, ** for $\mathrm{p}<0.01$, and ${ }^{* * * *}$ for $\mathrm{p}<0.001$.

\section{Additional files}

Additional file 1: Human endothelial cells were serum-starved $16 \mathrm{~h}(-)$ prior IL-8 challenge ( $+, 100 \mathrm{ng} / \mathrm{ml}, 15 \mathrm{~min})$. CXCR2 immunoprecipitation (IP) was performed as described in methods and IP fractions were blotted against CXCR2 and ubiquitination (Ub).

Additional file 2: Human THP1 monocytes were serum-starved $16 \mathrm{~h}$ (control) prior IL-8 challenge (+, $50 \mathrm{ng} / \mathrm{ml}, 15 \mathrm{~min})$. CXCR2 (Santa Cruz) and AP2 (Becton Dickinson) staining were analyzed by confocal microscopy. Scale bar: $10 \mu \mathrm{m}$

\section{Competing interests}

The authors declare that they have no competing interests.

\section{Authors' contributions}

HML: designed and executed the experiments, and interpreted the data; SMD: designed and executed the experiments, and interpreted the data; SA: designed and executed the experiments, and interpreted the data; JD: designed and executed the experiments, and interpreted the data; NB: designed and executed the experiments, and interpreted the data; JG: designed and executed the experiments, interpreted the data, and prepared the manuscript. All authors read and approved the final manuscript.

\begin{abstract}
Acknowledgements
The authors are thankful to the members of JG laboratory for comments on the manuscript. We are grateful to Thibaud de Chevigny, Jagoda K Hebda and Lucas Treps (Institut Cochin) for technical assistance and discussion, and to Mark GH Scott (Institut Cochin) for advice on $\beta$-arrestin2 experiments and to Alexandre Benmerah for DNA plasmids (Institut Necker, Paris, France). This research was funded by: Agence Nationale de la Recherche programme Jeunes Chercheuses et Jeunes Chercheurs, Fondation Association pour la Recherche sur le Cancer, Fondation pour la Recherche Medicale, Institut National du Cancer INCA_6508, Ligue nationale contre le cancer comite de Paris, Ligue nationale contre le cancer comite du Val-de-Marne. SMD is supported by a doctoral fellowship from Universite Paris Sud. SA and JD are supported by post-doctoral fellowship from Fondation Association pour la Recherche sur le Cancer and Agence Nationale de Recherche sur le SIDA et les hépatites virales, respectively.
\end{abstract}

\section{Author details}

${ }^{1}$ CNRS, UMR8104, 22 rue Mechain, 75014 Paris, France. ${ }^{2}$ INSERM, U1016, 22 rue Mechain, 75014 Paris, France. ${ }^{3}$ Universite Paris Descartes, Sorbonne Paris Cite, 12 rue de l'Ecole de Medecine, 75006 Paris, France. ${ }^{4}$ Institut Cochin, 22 rue Mechain, Rm. 306, 75014 Paris, France.
Received: 26 June 2014 Accepted: 9 October 2014

Published online: 22 October 2014

\section{References}

1. Addison CL, Daniel TO, Burdick MD, Liu H, Ehlert JE, Xue YY, Buechi L, Walz A, Richmond A, Strieter RM: The CXC chemokine receptor 2, CXCR2, is the putative receptor for ELR + CXC chemokine-induced angiogenic activity. $\mathrm{J}$ Immunol 2000, 165(9):5269-5277.

2. Dwyer J, Hebda JK, Le Guelte A, Galan-Moya EM, Smith SS, Azzi S, Bidere N, Gavard J: Glioblastoma cell-secreted interleukin-8 induces brain endothelial cell permeability via CXCR2. PLOS ONE 2012, 7(9):e45562.

3. Gavard J, Hou X, Qu Y, Masedunskas A, Martin D, Weigert R, Li X, Gutkind JS: A role for a CXCR2/phosphatidylinositol 3-kinase gamma signaling axis in acute and chronic vascular permeability. Mol Cell Biol 2009, 29(9):2469-2480.

4. Reutershan J: CXCR2-the receptor to hit? Drug News Perspect 2006, 19(10):615-623.

5. Koch AE, Polverini PJ, Kunkel SL, Harlow LA, DiPietro LA, Elner VM, Elner SG, Strieter RM: Interleukin-8 as a macrophage-derived mediator of angiogenesis. Science 1992, 258(5089):1798-1801.

6. Reutershan J, Morris MA, Burcin TL, Smith DF, Chang D, Saprito MS, Ley K: Critical role of endothelial CXCR2 in LPS-induced neutrophil migration into the lung. J Clin Invest 2006, 116(3):695-702.

7. Lazennec $G$, Richmond A: Chemokines and chemokine receptors: new insights into cancer-related inflammation. Trends Mol Med 2010, 16(3):133-144.

8. ljichi H, Chytil A, Gorska AE, Aakre ME, Bierie B, Tada M, Mohri D, Miyabayashi K, Asaoka Y, Maeda S, Ikenoue T, Tateishi K, Wright CV, Koike K, Omata M, Moses HL: Inhibiting Cxcr2 disrupts tumor-stromal interactions and improves survival in a mouse model of pancreatic ductal adenocarcinoma. $J$ Clin Invest 2011, 121(10):4106-4117.

9. Waugh DJJ, Wilson C: The Interleukin-8 Pathway in Cancer. Clin Cancer Res 2008, 14(21):6735-6741.

10. Cataisson C, Ohman R, Patel G, Pearson A, Tsien M, Jay S, Wright L, Hennings H, Yuspa SH: Inducible cutaneous inflammation reveals a protumorigenic role for keratinocyte CXCR2 in skin carcinogenesis. Cancer Res 2009, 69(1):319-328.

11. Dorsam RT, Gutkind JS: G-protein-coupled receptors and cancer. Nat Rev Cancer 2007, 7(2):79-94.

12. Marchese A, Trejo J: Ubiquitin-dependent regulation of $G$ protein-coupled receptor trafficking and signaling. Cell Signal 2013, 25(3):707-716.

13. Baugher PJ, Richmond A: The carboxyl-terminal PDZ ligand motif of chemokine receptor CXCR2 modulates post-endocytic sorting and cellular chemotaxis. J Biol Chem 2008, 283(45):30868-30878.

14. Fan GH, Lapierre LA, Goldenring JR, Richmond A: Differential regulation of CXCR2 trafficking by Rab GTPases. Blood 2003, 101(6):2115-2124.

15. Azzi S, Smith SS, Dwyer J, Leclair HM, Alexia C, Hebda JK, Dupin N, Bidere N, Gavard J: YGLF motif in the Kaposi sarcoma herpes virus G-proteincoupled receptor adjusts NF-kappaB activation and paracrine actions. Oncogene 2013. doi:10.1038/onc.2013.503.

16. Fan GH, Yang W, Wang XJ, Qian Q, Richmond A: Identification of a motif in the carboxyl terminus of CXCR2 that is involved in adaptin 2 binding and receptor internalization. Biochemistry 2001, 40(3):791-800.

17. Neel NF, Barzik M, Raman D, Sobolik-Delmaire T, Sai J, Ham AJ, Mernaugh $\mathrm{RL}$, Gertler FB, Richmond A: VASP is a CXCR2-interacting protein that regulates CXCR2-mediated polarization and chemotaxis. J Cell Sci 2009, 122(Pt 11):1882-1894.

18. Raman D, Sai J, Neel NF, Chew CS, Richmond A: LIM and SH3 protein-1 modulates CXCR2-mediated cell migration. PLOS ONE 2010, 5(4):e10050.

19. Raman D, Sai J, Hawkins O, Richmond A: Adaptor protein2 (AP2) orchestrates CXCR2-mediated cell migration. Traffic 2014, 15(4):451-469.

20. Dubois SM, Alexia C, Wu Y, Leclair HM, Leveau C, Schol E, Fest T, Tarte K, Chen ZJ, Gavard J, Bidere N: A catalytic-independent role for the LUBAC in NF-kappaB activation upon antigen receptor engagement and in lymphoma cells. Blood 2014, 123(14):2199-2203.

21. Oeckinghaus A, Wegener E, Welteke V, Ferch U, Arslan SC, Ruland J, Scheidereit C, Krappmann D: Malt1 ubiquitination triggers NF-kappaB signaling upon T-cell activation. EMBO J 2007, 26(22):4634-4645.

22. White JR, Lee JM, Young PR, Hertzberg RP, Jurewicz AJ, Chaikin MA, Widdowson K, Foley JJ, Martin LD, Griswold DE, Sarau HM: Identification of a potent, selective non-peptide CXCR2 antagonist that inhibits 
interleukin-8-induced neutrophil migration. J Biol Chem 1998, 273(17):10095-10098.

23. Lefkowitz RJ: Historical review: a brief history and personal retrospective of seven-transmembrane receptors. Trends Pharmacol Sci 2004, 25(8):413-422.

24. Hebda JK, Leclair HM, Azzi S, Roussel C, Scott MG, Bidere N, Gavard J: The C-terminus region of beta-arrestin 1 modulates VE-cadherin expression and endothelial cell permeability. Cell Commun Signal 2013, 11(1):37.

25. Rappoport JZ, Taha BW, Lemeer S, Benmerah A, Simon SM: The AP-2 complex is excluded from the dynamic population of plasma membrane-associated clathrin. J Biol Chem 2003, 278(48):47357-47360.

26. Lakadamyali M, Rust MJ, Zhuang X: Ligands for clathrin-mediated endocytosis are differentially sorted into distinct populations of early endosomes. Cell 2006, 124(5):997-1009.

27. Wu S, Zhang Y: LOMETS: a local meta-threading-server for protein structure prediction. Nucleic Acids Res 2007, 35(10):3375-3382.

28. Gavard J, Gutkind JS: Protein kinase C-related kinase and ROCK are required for thrombin-induced endothelial cell permeability downstream from Galpha12/13 and Galpha11/q. J Biol Chem 2008, 283(44):29888-29896.

29. Charest PG, Terrillon S, Bouvier M: Monitoring agonist-promoted conformational changes of beta-arrestin in living cells by intramolecular BRET. EMBO Rep 2005, 6(4):334-340.

doi:10.1186/s12860-014-0038-0

Cite this article as: Leclair et al: Control of CXCR2 activity through its ubiquitination on K327 residue. BMC Cell Biology 2014 15:38.

\section{Submit your next manuscript to BioMed Central and take full advantage of:}

- Convenient online submission

- Thorough peer review

- No space constraints or color figure charges

- Immediate publication on acceptance

- Inclusion in PubMed, CAS, Scopus and Google Scholar

- Research which is freely available for redistribution 